\title{
On the Isomorphism Problem for Decision Trees and Decision Lists ${ }^{\star}$
}

\author{
V. Arvind ${ }^{1}$, Johannes Köbler ${ }^{2}$, Sebastian Kuhnert ${ }^{2}$, \\ Gaurav Rattan ${ }^{1}$, and Yadu Vasudev ${ }^{1}$ \\ 1 The Institute of Mathematical Sciences, Chennai, India \\ \{arvind, grattan, yadu\}@imsc.res.in \\ 2 Institut für Informatik, Humboldt-Universität zu Berlin, Germany \\ \{koebler, kuhnert\}@informatik.hu-berlin.de
}

\begin{abstract}
We study the complexity of isomorphism testing for Boolean functions that are represented by decision trees or decision lists. Our results include a $2^{\sqrt{s}(\lg s)^{O(1)}}$ time algorithm for isomorphism testing of decision trees of size $s$. Additionally, we show:

- Isomorphism testing of rank-1 decision trees is complete for logspace.

- For $r \geq 2$, isomorphism testing for rank- $r$ decision trees is polynomialtime equivalent to Graph Isomorphism. As a consequence we obtain a $2^{\sqrt{s}(\lg s)^{O(1)}}$ time algorithm for isomorphism testing of decision trees of size $s$.

- The isomorphism problem for decision lists admits a Schaefer-type dichotomy: depending on the class of base functions, the isomorphism problem is either in polynomial time, or equivalent to Graph Isomorphism, or coNP-hard.
\end{abstract}

\section{Introduction}

Two Boolean functions $f, g:\{0,1\}^{n} \rightarrow\{0,1\}$ are said to be isomorphic if there is a permutation $\pi$ of the input variables $x_{1}, x_{2}, \ldots, x_{n}$ so that $f\left(x_{1}, x_{2}, \ldots, x_{n}\right)$ and $g\left(x_{\pi(1)}, x_{\pi(2)}, \ldots, x_{\pi(n)}\right)$ are equivalent Boolean functions. The Boolean function isomorphism problem is to test if two given Boolean functions $f$ and $g$ are isomorphic. The complexity of this problem, when $f$ and $g$ are given as either Boolean circuits, formulas, or branching programs, has been studied before AT96. The isomorphism problem for Boolean circuits is in $\Sigma_{2}^{p}$ and is coNP-hard even for DNF formulas. It is also known [AT96] that the problem is not hard for $\Sigma_{2}^{p}$ unless the polynomial hierarchy collapses. Thierauf Thi00 further studied isomorphism and equivalence for various models of Boolean functions. He has shown that the isomorphism problem for read-once branching programs is not NP-complete unless the polynomial hierarchy collapses to $\Sigma_{2}^{p}$. From an algorithmic perspective, Boolean Function Isomorphism can be solved in $2^{O(n)}$ time [Luk99] by reducing it to Hypergraph Isomorphism, and this is the best known algorithm in general. The

\footnotetext{
* This work was supported by Alexander von Humboldt Foundation in its research
} group linkage program. The third author was supported by DFG grant KO 1053/7-1. 
best known algorithm for Graph Isomorphism, on the other hand, has running time $2^{O(\sqrt{n \lg n})}$ BL83.

In this paper, our aim is to explore Boolean function representations for which the isomorphism problem has faster algorithms. We focus on the problem when the functions are given as decision trees and decision lists.

Definition 1.1. A decision tree $T_{f}$ on variables $X=\left\{x_{1}, \ldots, x_{n}\right\}$ is an ordered binary tree in which each leaf is labeled with a Boolean value and each inner node is labeled with a variable in $X$ and has exactly two children. Any assignment $b_{1}, \ldots, b_{n}$ defines a path from the root of $T_{f}$ to a leaf: At an inner node labeled with $x_{i}$, proceed to the left child if $b_{i}=0$ and to the right child otherwise. The function value $T_{f}\left(b_{1}, \ldots, b_{n}\right)$ is the label of the leaf node reached along this path.

Decision trees are a natural representation for Boolean functions and are fundamental to Boolean function complexity. The size of a decision tree is the number of its leaves.

The satisfiability and equivalence problems for decision trees have simple polynomial-time algorithms. Thus, the isomorphism problem for decision trees, denoted DT-Iso, is in NP.

Our main result is a $2^{\sqrt{s}(\lg s)^{O(1)}}$ time algorithm for isomorphism testing of size- $s$ decision trees. We obtain this algorithm by examining the connection between bounded rank decision trees and hypergraphs of bounded rank. The rank of a hypergraph is the maximum hyperedge size in the hypergraph, and the rank of a decision tree $T$ is the depth of the largest full binary tree that can be embedded in $T$. It turns out that rank- $r$ decision trees can be encoded as hypergraphs of rank $O(r)$ and this transformation can be carried out in time $n^{O(r)}$. Since decision trees of size $s$ have rank at most $\lg s$, this gives the $2^{\sqrt{s}(\lg s)^{O(1)}}$ time algorithm for isomorphism by applying the algorithm for bounded rank Hypergraph Isomorphism described in [BC08. Further, it turns out that isomorphism of rank-1 decision trees is complete for deterministic logspace.

The next main topic of the paper is the isomorphism problem for decision lists, which were originally introduced by Rivest [Riv87] in learning theory.

Definition 1.2 ([Riv87]). A $\mathcal{C}$-decision list $(\mathcal{C}-D L) L$, where $\mathcal{C}$ is a class of Boolean functions, is a sequence of the form $\left(f_{i}, b_{i}\right)_{i \leq m}$ where $f_{i} \in \mathcal{C}, b_{i} \in\{0,1\}$ and $f_{m}=1$. For a Boolean assignment $x$, the value computed by the decision list $L(x)$ is defined as $b_{i}$, where $i=\min \left\{j \geq 1 \mid f_{j}(x)=1\right\}$.

If $\mathcal{C}$ consists of single literals then $\mathcal{C}$-DL coincides with rank-1 decision trees. Similarly, if $\mathcal{C}$ consists of conjunctions of $r$ literals then every $r$-CNF or $r$-DNF formula has a $\mathcal{C}$-decision list. We call such decision lists $r$-decision lists (or $r$-DLs, in short). For $r \geq 3$, the satisfiability problem for $r$-DLs is clearly NPcomplete, and the equivalence problem is coNP-complete. Furthermore, every rank- $r$ decision tree of size $s$ has an $r$-decision list of size $O(s)$. Our results on isomorphism testing for decision lists are summarized below. We restrict our attention to classes $\mathcal{C}$ of Boolean functions depending only on at most $k$ variables, for constant $k$. 
1. If $\mathcal{C}$ consists of parities on 2 literals, isomorphism testing for $\mathcal{C}$-DLs is in polynomial time.

2. Isomorphism testing for $\mathcal{C}$-DLs is $\mathrm{Gl}$-complete ${ }^{3}$ when $\mathcal{C}$ is one of the following: (i) the conjunction of two literals, (ii) a conjunction of literals with at most one negative literal, (iii) a conjunction of literals with at most one positive literal, and (iv) parities on three or more literals.

3. In all other cases for $\mathcal{C}$, isomorphism testing for $\mathcal{C}$-DLs is coNP-hard.

The above results show a Schaefer-type dichotomy for the $\mathcal{C}$-DL isomorphism problem. It is interesting to compare with the dichotomy results for $\mathcal{C}$-CSP isomorphism obtained by Böhler et al. [BHRV04. In their paper, the dichotomy exactly corresponds to Schaefer's original classification [Sch78]. In our results above, we have the Boolean complements of the Schaefer classes.

We observe that any $\mathcal{C}$-CSP $F=C_{1} \wedge C_{2} \wedge \ldots \wedge C_{s}$ is equivalent to the $\mathcal{C}$-DL given by $L=\left(\neg C_{1}, 0\right), \ldots,\left(\neg C_{s}, 0\right),(1,1)$, proving the following lemma.

Lemma 1.3. Let $\mathcal{C}$ be any class of Boolean functions closed under negation. Given a $\mathcal{C}$-CSP $F$ of size $s$, there is a $\mathcal{C}$-decision list $L$ of size $s+1$ that is equivalent to $F$.

We now recall the notion of rank for decision trees [EH89. Let $T$ be a decision tree and $v$ be a node in $T$. If $v$ is a leaf node then its $\operatorname{rank} \operatorname{isk}(v)=0$. Otherwise, suppose $v$ has children $v_{1}$ and $v_{2}$ in $T$. If $\operatorname{rk}\left(v_{1}\right) \neq \operatorname{rk}\left(v_{2}\right)$, define $\operatorname{rk}(v)=\max \left\{\operatorname{rk}\left(v_{1}\right), \operatorname{rk}\left(v_{2}\right)\right\} ;$ if $\operatorname{rk}\left(v_{1}\right)=\operatorname{rk}\left(v_{2}\right)$, define $\operatorname{rk}(v)=\operatorname{rk}\left(v_{1}\right)+1$. The rank of the decision tree $\operatorname{rk}(T)$ is the rank of its root node. The $\operatorname{rank} \operatorname{rk}(f)$ of a Boolean function $f$ is the minimum rank over all the decision trees computing $f$.

In general, by a representation of a Boolean function $f:\{0,1\}^{n} \rightarrow\{0,1\}$ we mean a finite description $R$ for $f$, such that for any input $x \in\{0,1\}^{n}$ we can evaluate $R(x)=f(x)$ in time polynomial in $n$. Examples of representations include circuits, branching programs, formulas, decision trees, decision lists etc.

Let $\pi$ be a permutation of the input variables $x_{1}, x_{2}, \ldots, x_{n}$. Then $f^{\pi}$ denotes the Boolean function $f\left(x_{\pi(1)}, x_{\pi(2)}, \ldots, x_{\pi(n)}\right)$. Similarly, for any representation $R$ of the function $f$ we denote by $R^{\pi}$ the representation for $f^{\pi}$ obtained by replacing each input variable $x_{i}$ in $R$ by $x_{\pi(i)}$.

Let $\mathcal{R}$ and $\mathcal{R}^{\prime}$ be sets of representations of Boolean functions. A permutation preserving normal form representation (in short, normal form) for $\mathcal{R}$ is a mapping $N: \mathcal{R} \rightarrow \mathcal{R}^{\prime}$ such that (i) for any $R \in \mathcal{R}, N_{R}$ and $R$ describe the same function, (ii) if $R_{1}$ and $R_{2}$ describe the same function then $N_{R_{1}}=N_{R_{2}}$, and (iii) for each permutation $\pi$ we have $N_{R^{\pi}}=\left(N_{R}\right)^{\pi}$.

A canonical form representation for $\mathcal{R}$ is a mapping $C: \mathcal{R} \rightarrow \mathcal{R}^{\prime}$ such that (i) for any $R \in \mathcal{R}$, the function represented by $C_{R}$ is isomorphic to the one described by $R$, and (ii) for any two representations $R_{1}$ and $R_{2}$, the functions described by $R_{1}$ and by $R_{2}$ are isomorphic if and only if $C_{R_{1}}=C_{R_{2}}$.

Suppose $f$ is a rank-r Boolean function, and $f$ is given as a decision tree $T_{f}$ which is not necessarily of rank $r$. There is a recursive $n^{O(r)}$ time algorithm for

\footnotetext{
${ }^{3}$ We say that a decision problem is $\mathrm{Gl}$-complete if it is polynomial-time equivalent to Graph Isomorphism.
} 
computing a rank- $r$ decision tree for $f$. This procedure is useful in isomorphism testing for bounded rank decision trees. As base case, suppose $f$ is rank 1 and is given by $T_{f}$. Then there is a variable $x$ such that $\left.f\right|_{x \leftarrow 0}$ or $\left.f\right|_{x \leftarrow 1}$ is constant, where $\left.f\right|_{x \leftarrow b}$ denotes $f$ with $x$ set to $b$. This can be checked by setting $x=b$ in $T_{f}$ and verifying that all leaves in the modified decision tree are labeled by the same constant. Suppose $x_{i}$ is a variable such that $\left.f\right|_{x_{i} \leftarrow 0}$ is the constant function 1 , then the function $\left.f\right|_{x_{i} \leftarrow 1}$ is of rank 1 and has only $n-1$ variables. Proceeding thus, in time polynomial in the size of $T_{f}$ we can check if $f$ is of rank 1 and also compute a rank-1 decision tree for it. For checking if $f$ has rank $r$, we sketch a simple recursive procedure: Find a variable $x_{i}$ such that $\left.f\right|_{x \leftarrow b}$ is of rank at most $r-1$ (checked recursively), where $b \in\{0,1\}$. If $\left.f\right|_{x \leftarrow \bar{b}}$ has rank at most $r$ (checked recursively) then $f$ is of rank $r$, else $f$ has rank more than $r$. If no such variable exists then $f$ is not rank $r$. The correctness and running time bounds are straightforward by induction arguments.

Theorem 1.4. Given as input a decision tree $T$, we can check if the computed Boolean function has rank $r$ and, if so, construct a rank-r decision tree for it in time $\left(n^{r} \cdot|T|\right)^{O(1)}$.

\section{GI-Hardness of DT-Iso and $\mathcal{C}$-DL-Iso}

We will show that isomorphism testing even for rank-2 decision trees is GI-hard.

Let $G=(V, E)$ be a graph with $V=\left\{v_{1}, v_{2} \ldots, v_{n}\right\}$ and $E=\left\{e_{1}, e_{2}, \ldots, e_{m}\right\}$. We encode $G$ as a Boolean function $f_{G}$ on $n+m$ Boolean variables $v_{1}, \ldots, v_{n}$ and $e_{1}, \ldots, e_{m}$ as follows: $f_{G}\left(e_{1}, \ldots, e_{m}, v_{1}, \ldots, v_{n}\right)=1$ if and only if exactly three variables $e_{i}, v_{j}, v_{k}$ are 1 , all remaining variables are 0 , and $e_{i}=\left(v_{j}, v_{k}\right) \in E$. Here the Boolean variables $v_{i}$ and $e_{j}$ correspond, by abuse of notation, to elements of $V \cup E$. The proofs for the following simple observations are omitted for lack of space.

Lemma 2.1. For any graph $G(V, E)$, the function $f_{G}$ is of rank 2 and can be represented by a rank-2 decision tree of size $O\left(|G|^{2}\right)$.

Theorem 2.2. Let $G$ and $H$ be two graphs and let $f_{G}$ and $f_{H}$ be the functions as defined above. Then, $G \cong H$ if and only if $f_{G} \cong f_{H}$.

Corollary 2.3. GI $\leq_{p}^{m}$ DT-Iso.

We now give a simple reduction from Graph Isomorphism to 2-DNF Isomorphism. Since $\mathcal{C}$-DLs, where $\mathcal{C}$ is the class of conjunctions of 2 literals or of $k$ literals with at most 1 negative literal, contains 2-DNFs Riv87, this will prove $\mathcal{C}$-DL-Iso is $\mathrm{Gl}$-hard for this choice of $\mathcal{C}$.

Given a graph $G(V, E)$, define the following functions over the variable set $V$ : $\check{f}_{G}=\bigvee_{e=(u, v) \in E} u \wedge v, \hat{f}_{G}=\bigvee_{e=(u, v) \in E} \bar{u} \wedge \bar{v}$.

Lemma 2.4. Let $G, H$ be two graphs. Then $G \cong H$ if and only if $\check{f}_{G} \cong \check{f}_{H}$ if and only if $\hat{f}_{G} \cong \hat{f}_{H}$. 
Böhler et al. BHRV04 have considered $\mathcal{C}$-CSP Isomorphism, where the constraints in $\mathcal{C}$ are all XORs of $k$ literals for constant $k \geq 3$. They have shown that this problem is Gl-hard. Combined with Lemma 1.3 and Lemma 2.4 this yields the following.

Proposition 2.5. GI $\leq_{p}^{m} \mathcal{C}$-DL-Iso, where $\mathcal{C}$ consists either of (i) conjunctions of two literals, (ii) conjunctions of $k$ literals with at most one positive literal each, (iii) conjunctions of $k$ literals with at most one negative literal each or (iv) XORs of $k \geq 3$ literals, for some constant $k$.

In Section 4 we will show that these problems are Gl-complete.

\section{$3 \quad$ Isomorphism for Bounded Rank Decision Trees}

We first show that the isomorphism problem for rank-1 Boolean functions is in polynomial time. In fact, we will give a polynomial-time algorithm for computing a canonical form representation for rank-1 Boolean functions. If the rank-1 function is given as a rank-1 decision tree, we show that the isomorphism problem is complete for deterministic logspace. Building on the rank-1 case, we give a polynomial-time reduction from the isomorphism problem for bounded rank Boolean functions to isomorphism of bounded rank hypergraphs. This yields a moderately exponential time algorithm for isomorphism testing of bounded rank decision trees.

Let $f$ be a rank-1 Boolean function given by some decision tree $T_{f}$ which is not necessarily rank 1 .

Since $\operatorname{rk}(f)=1$, for some variable $x_{i}$ the function $\left.f\right|_{x_{i} \leftarrow b}$ is a constant. Let $V_{1}(f)$ be the subset of variables $x_{i} \in\left\{x_{1}, x_{2}, \ldots, x_{n}\right\}$ such that $\left.f\right|_{x_{i} \leftarrow 1}$ or $\left.f\right|_{x_{i} \leftarrow 0}$ is a constant function. We define two subsets of $V_{1}(f): V_{1,0}(f)=\left\{x_{i} \mid\right.$ $\left.f\right|_{x_{i} \leftarrow 0}$ is constant $\}$ and $V_{1,1}(f)=\left\{x_{i}|f|_{x_{i} \leftarrow 1}\right.$ is constant $\}$. The set $V_{1}(f)$ is computable in polynomial time from $T_{f}$ : to check if $x_{i} \in V_{1}(f)$ we fix $x_{i}$ to a constant in $T_{f}$ and see if all leaves in the resulting decision tree have the same label.

In general, if $T$ is a decision tree computing a function $f, x_{i}$ is some variable and $b$ is a Boolean constant, we can obtain the decision tree $\left.T\right|_{x_{i} \leftarrow b}$ for the function $\left.f\right|_{x_{i} \leftarrow b}$ by removing the subtree corresponding to the $\bar{b}$ path of any node labeled with the variable $x_{i}$. To check if this is the constant function, it is enough to verify if all the leaves of this modified decision tree have the same constant.

Next, we define $f_{0}=f$ and $f_{i}=\left.f_{i-1}\right|_{V_{1,0}\left(f_{i-1}\right) \leftarrow 1, V_{1,1}\left(f_{i-1}\right) \leftarrow 0}$. We also define the variable sets $V_{i}(f)=V_{1}\left(f_{i-1}\right)$, which are again classified into $V_{i, 0}(f)=$ $V_{1,0}\left(f_{i-1}\right)$ and $V_{i, 1}(f)=V_{1,1}\left(f_{i-1}\right)$. The variable set $\left\{x_{1}, x_{2}, \ldots, x_{n}\right\}$ is thus partitioned into $V_{1}(f), \ldots, V_{k}(f)$ for some $k \leq n$. The level of a variable $x_{i}$ is the index $j$ such that $x_{i} \in V_{j}(f)$.

The normal form for the rank-1 function $f$ is defined as the sequence of pairs $\left\langle l_{i}, c_{i}\right\rangle_{1 \leq i \leq r}$ where $l_{i}$ is a variable or its complement, and $c_{i} \in\{0,1\}$. The pairs $\left\langle l_{i}, c_{i}\right\rangle$ in the sequence are ordered from left to right in increasing order of variable levels. Within each level they are in increasing order of variable 
indices. For $x_{i} \in V_{j, 0}(f)$, add $\left\langle\overline{x_{i}},\left.f_{j-1}\right|_{x_{i} \leftarrow 0}\right\rangle$ to the sequence; for $x_{i} \in V_{j, 1}(f)$, add $\left\langle x_{i},\left.f_{j-1}\right|_{x_{i} \leftarrow 1}\right\rangle$ to the sequence.

Suppose $f$ is a rank 1 function given by a decision tree $T$. Let the above sequence computed from $T$ be $N_{T}$ (which is actually a decision list for $f$ ). This sequence $N_{T}$ defines a normal form representation for $T$. This follows from the next two lemmas.

Lemma 3.1. Suppose $f$ and $g$ are two isomorphic rank-1 Boolean functions and $\pi$ is an isomorphism from $f$ to $g$. For any input variable $x$, if $x$ is in level $j$ for $f$ then variable $\pi(x)$ is in level $j$ for $g$.

This implies that the number of variables in each level coincides for two isomorphic Boolean functions of rank 1. The next lemma is in the converse direction.

Lemma 3.2. Let $T_{1}$ and $T_{2}$ be decision trees for two Boolean functions of rank 1 , defined on the $n$ variables $x_{1}, \ldots, x_{n}$. Let $N_{T_{1}}$ and $N_{T_{2}}$ be the corresponding normal form sequences obtained as in the discussion above. Suppose for each level $i$, $\left|V_{i, 0}(f)\right|=\left|V_{i, 0}(g)\right|$ and $\left|V_{i, 1}(f)\right|=\left|V_{i, 1}(g)\right|$, then $T_{1}$ and $T_{2}$ are isomorphic.

Hence, the defined sequences are normal forms for rank-1 functions and in time polynomial in the size of the input decision tree we can compute the normal form. Given $T_{1}$ and $T_{2}$, Lemma 3.2 shows that by comparing the sizes of the sets $V_{i, 0}$ and $V_{i, 1}$ for the two functions we can check if the Boolean functions are isomorphic or not. This gives us the following theorem.

Theorem 3.3. Given Boolean functions of rank 1 by decision trees, there is a polynomial time algorithm that checks if the functions are isomorphic.

We now show that if the rank- 1 function $f$ is given as a decision tree $T_{f}$ which is of rank 1, then the canonization problem is in logspace. For each internal node of $T_{f}$ at least one of its children is a leaf (labeled by a constant). We can partition the internal nodes in the tree into subsets $L_{1}, \ldots, L_{m}$, where $L_{1}$ has consecutive nodes starting from the root that have a leaf child labelled with the same Boolean constant as at the root, $L_{2}$ is the next set of consecutive nodes with a leaf child labelled with the Boolean constant opposite to the one at the root, and so on. We further classify the variables in each $L_{i}$ into the subset $L_{i, 0}$ of nodes in $L_{i}$ whose left child is a leaf and subset $L_{i, 1}$ of nodes whose right child is a leaf. These sets can be computed in logspace by inspection of the input decision tree. Since for each $x \in L_{1, b}$, the restriction $\left.f\right|_{x \leftarrow b}$ is a constant, $L_{1, b} \subseteq V_{1, b}(f)$. Also, notice that no variable outside the set $L_{1, b}$ has this property. Hence $L_{1, b}=V_{1, b}(f)$. Likewise, we can argue that $L_{i, b}=V_{i, b}(f)$ for all $i$ and $b$. As a consequence we can state the following lemma.

Lemma 3.4. Let $T$ and $T^{\prime}$ be two rank-1 decision trees computing equivalent Boolean functions. Then $L_{i, b}=L_{i, b}^{\prime}$ for all $i$ and $b$.

In order to obtain the canonical form for rank-1 decision trees, we first order the variables of $L_{i}$ such that all the nodes whose left child is a constant come 
first followed by the nodes whose right child is a constant. We do this for all the sets $L_{1}, \ldots, L_{m}$. Now starting from the root, rename the variables with the root node getting the variable $x_{1}$ followed by $x_{2}$ and so on. This new decision tree $T_{c}$, which is clearly computable in logspace, will be the canonical form for the original decision tree. By Lemma 3.4 a rank-1 decision tree isomorphic to the given tree is obtained by permuting the variables in $L_{i}$ in some way for each $i$. Hence given two rank-1 decision trees computing isomorphic Boolean functions, the above procedure outputs the same rank- 1 decision tree proving that it is a canonical form.

We now show the logspace completeness. We will give a reduction from the problem PathCenter, which is known to be complete for $\mathrm{L}$ ADKK12. The input to PathCenter is a directed path $P$ of odd length and a vertex $u$. The problem is to test if $u$ is the center of the path. We construct two decision trees $T_{1}$ and $T_{2}$ from $P$ : For each $v \in V$ there is a variable $x_{v}$, and both $T_{1}$ and $T_{2}$ contain one internal node for each $x_{v}$. If $v$ is the successor of $v^{\prime}$ in $P, x_{v}$ becomes the right child of $x_{v^{\prime}}$ in $T_{1}$, and $x_{v^{\prime}}$ becomes the right child of $x_{v}$ in $T_{2}$. The right child of $x_{v}$, where $v$ is the vertex without successor (or without predecessor, respectively) is a leaf labeled with 1 . In both trees, the left child of $x_{u}$ is a leaf labeled with 1 . The left children of all other variables are leaves labeled with 0 .

Lemma 3.5. Let $T_{1}$ and $T_{2}$ be the decision trees constructed from an instance $(P(V, E), u)$ of PathCenter. Let $f_{1}$ and $f_{2}$ be the functions computed by the decision trees, respectively. Then, $f_{1} \cong f_{2}$ if and only if $(P(V, E), u) \in$ PathCenter.

We now consider rank- $r$ Decision Tree Isomorphism. Using the normal form representation for rank-1 Boolean functions, we will obtain normal forms for Boolean functions of rank $r$. Similar to rank-1 decision trees, where the normal form consists of literal and constant pairs, for bounded rank functions the normal form will consist of pairs of literals and a normal form of a rank $r-1$ Boolean function. Let $V_{1}(f)$ be the subset of variables $x_{i}$ such that $\left.f\right|_{x_{i} \leftarrow 0}$ or $\left.f\right|_{x_{i} \leftarrow 1}$ has rank at most $r-1$. Let $V_{1, b}(f) \subseteq V_{1}(f)$ consist of $x_{i}$ such that $\left.f\right|_{x_{i} \leftarrow b}$ has rank at most $r-1$ for $b \in\{0,1\}$. Further partition $V_{1, b}$ into subsets $V_{1, b}^{\ell}$ for $1 \leq \ell \leq r-1$ where $V_{1, b}^{\ell}=\left\{x_{i} \in V_{1}(f)|f|_{x_{i} \leftarrow b}\right.$ has rank $\left.\ell\right\}$.

For each $x_{i} \in V_{1,0}^{\ell}$ in increasing order of index $i$, include the pair $\left\langle\overline{x_{i}}, N_{x_{i}}\right\rangle$ in the sequence, where $N_{x_{i}}$ is the normal form for $\left.f\right|_{x_{i} \leftarrow 0}$, defined recursively. Similarly for $x_{i} \in V_{1,1}^{\ell}$ in the increasing order of the variable name, add the tuple $\left\langle x_{i}, N_{x_{i}}\right\rangle$ to the sequence. The above procedure is carried out for $\ell$ increasing from 1 to $r-1$. Now define, $f_{1}=\left.f\right|_{V_{1,0}(f) \leftarrow 1, V_{1,1}(f) \leftarrow 0}$, and continue constructing the normal form for $f_{1}$ as explained above. In general, we define the variable subsets $V_{i, b}^{\ell}(f)=V_{1, b}^{\ell}\left(f_{i-1}\right)$. Since for each $x_{i}$, checking if $\left.f\right|_{x_{i} \leftarrow b}$ is a rank- $\ell$ function and to compute it takes poly $\left(n^{r-1}|T|\right)$ time by Theorem 1.4, and since this process has to be repeated for at most $n$ steps, the normal form can be constructed in time poly $\left(n^{r}|T|\right)$. The normal form consists of a sequence $\left\langle l_{i}, N_{x_{i}}\right\rangle_{i \leq m}$ where $m \leq n$ and $l_{i} \in\left\{x_{i}, \overline{x_{i}}\right\}$. We summarize the discussion in the following lemma (without proof). 
Lemma 3.6. Given a decision tree $T$ computing a Boolean function $f$ of rank $r$, a normal form representation $N_{T}$ for the function can be computed in time $\operatorname{poly}\left(n^{r}|T|\right)$.

We now describe our reduction of rank- $r$ Decision Tree Isomorphism to bounded rank Hypergraph Isomorphism, where the rank of a hypergraph is the maximum size of any hyperedge in it.

Given a rank- $r$ Boolean function as a decision tree, we first construct the normal form for $f, N_{f}$ in time $n^{O(r)}$ as described earlier. The next step is to construct a vertex-colored hypergraph corresponding to the normal form. We will encode all the information in the normal form using hyperedges. The construction is inductive.

Rank-1 functions: The case of rank- 1 functions is easy, since the normal form for a rank-1 Boolean function consists of a decision tree where for each node, one of its children is a constant. In the hypergraph corresponding to the rank-1 function $f$, for each variable $x_{i}$ that appears in the normal form we add a vertex $v_{i}$. Add the vertices $(i, b)$ where $1 \leq i \leq n$ and $b \in\{0,1\}$. We also add two vertices $\mathbf{0}$ and $\mathbf{1}$ corresponding to the constants. Now for each variable $x_{i}$, if $x_{i} \in V_{j, b}(f)$ and one of its children is labeled with the constant $c$, add the hyperedge $\left(v_{i},(j, b), c\right)$ in the hypergraph. We color all the vertices corresponding to the variables with one color and each $(j, b)$ with a separate color. The vertices $\mathbf{0}$ and $\mathbf{1}$ are colored with different colors as well. Call the resulting rank-3 hypergraph $\mathcal{H}_{f}$. We have the following lemma.

Lemma 3.7. Let $f$ and $g$ be Boolean functions of rank 1 given by decision trees, and let $\mathcal{H}_{f}$ and $\mathcal{H}_{g}$ be the hypergraphs constructed as above. Then, $f$ and $g$ are isomorphic as functions if and only if the hypergraphs $\mathcal{H}_{f}$ and $\mathcal{H}_{g}$ are isomorphic.

Rank-r functions: Let $f$ be a rank-r function, and let $N_{f}=\left\langle l_{i}, N_{x_{i}}\right\rangle_{i \leq k}$, where $k \leq n$ and $l_{i} \in\left\{x_{i}, \overline{x_{i}}\right\}$, be the normal form for $f$. The vertex set for the hypergraph $\mathcal{H}_{f}$ is $\left\{u_{1}, \ldots, u_{n}\right\} \cup\left\{v_{1}^{d}, \ldots, v_{n}^{d} \mid 1 \leq d \leq r\right\} \cup\{(l, i, b, j) \mid 1 \leq$ $l \leq r, 1 \leq i \leq n, 1 \leq j \leq r, b \in\{0,1\}\} \cup\{\mathbf{0}, \mathbf{1}\}$. Intuitively, the vertices $u_{1}, \ldots, u_{n}$ will encode the variables $x_{1}, \ldots, x_{n}$ and $v_{1}^{1}, \ldots, v_{n}^{1}$ will encode the variables $x_{1}, \ldots, x_{n}$ at the outermost level in $\left(l_{1}, N_{x_{i}}\right)$ pairs. Let $\mathcal{H}_{i}$ denote the hypergraph encoding $N_{x_{i}}$, constructed inductively. The vertex set of $\mathcal{H}_{i}$ will be $\left\{v_{1}^{d}, \ldots, v_{n}^{d} \mid 2 \leq d \leq r\right\} \cup\{(l, i, b, j) \mid 1 \leq l \leq r-1,1 \leq i \leq n, b \in\{0,1\}$, $2 \leq j \leq r\} \cup\{\mathbf{0}, \mathbf{1}\}$. We define the edge set for $\mathcal{H}_{f}$ as follows: For every $\left\langle l_{i}, N_{x_{i}}\right\rangle$ in the normal form and every edge $e \in \mathcal{H}_{i}$, we include $e \cup\left\{v_{i}^{1}\right\} \cup\{(l, j, b, 1)\}$ in the edge set if $x_{i} \in V_{j, b}^{l}(f)$ (where $b$ encodes whether $l_{i}$ is $x_{i}$ or $\overline{x_{i}}$ ) and the edges $\left\{u_{i}, v_{i}^{1}\right\}$ for all $i$. Assume, inductively, that $\mathcal{H}_{i}$ is of rank at most $2(r-1)+1$. Then clearly $H_{f}$ is of rank at most $2 r+1$. If $f \cong g$ via $\pi \in S_{n}$, then since $N_{f}$ and $N_{g}$ are their normal form representations $\left(N_{f}\right)^{\pi}=N_{g}$, where $\left(N_{f}\right)^{\pi}$ is obtained by replacing $x_{i}$ by $x_{\pi(i)}$ for all $i$ in $N_{f}$. By induction on the rank $r$, we can easily argue that there is a $\pi \in S_{n}$ such that $\left(N_{f}\right)^{\pi}=N_{g}$ if and only if the hypergraphs $H_{f}$ and $H_{g}$ are isomorphic. 
Lemma 3.8. Let $f$ and $g$ be Boolean functions of rank $r$ given by decision trees. Let $\mathcal{H}_{f}$ and $\mathcal{H}_{g}$ be the hypergraphs constructed as above. Then, $f$ and $g$ are isomorphic as functions if and only if the hypergraphs $\mathcal{H}_{f}$ and $\mathcal{H}_{g}$ are isomorphic.

According to the construction, the hypergraph $\mathcal{H}_{f}$ corresponding to the rank- $r$ function $f$ has $2 n r$ vertices and rank $2 r+1$. The size of the hypergraph is at most $n^{O(r)}$ since any rank- $r$ Boolean function has a rank- $r$ decision tree of size $n^{O(r)}$. In particular, the normal form that we construct is of size at most $n^{O(r)}$. We formulate these observations in the following theorem.

Theorem 3.9. Let $f$ and $g$ be Boolean functions of rank $r$ given by decision trees $T_{f}$ and $T_{g}$. There is an algorithm running in time $n^{O(r)}$ that outputs two hypergraphs $\mathcal{H}_{f}$ and $\mathcal{H}_{g}$ of rank $2 r+1$ and size $n^{O(r)}$ such that $f$ and $g$ are isomorphic if and only if the hypergraphs $\mathcal{H}_{f}$ and $\mathcal{H}_{g}$ are isomorphic.

Since any decision tree of size $s$ has rank at most $O(\log s)$, it has a normal form representation of size $n^{O(\log s)}$ which can be computed in $n^{O(\log s)} \cdot s^{O(1)}$. Hence we have the following corollary.

Corollary 3.10. Let $f$ and $g$ be two decision trees of size $s$. There is an $s^{O(\log s)}$ time algorithm which computes hypergraphs $\mathcal{H}_{f}$ and $\mathcal{H}_{g}$ of logarithmic rank and size $s^{O(\log s)}$ such that $f$ and $g$ are isomorphic if and only if $\mathcal{H}_{f} \cong \mathcal{H}_{g}$.

Combining this with the isomorphism algorithm for hypergraphs of bounded rank due to Babai and Codenotti [BC08], we observe the following:

Corollary 3.11. Given two Boolean functions $f$ and $g$ as decision trees of size $s$, there is a $2^{\sqrt{s}(\log s)^{O(1)}}$ time algorithm to check if $f \cong g$.

\section{Isomorphism for Decision Lists}

We now consider $\mathcal{C}$-DL Isomorphism (defined in Section 1), where $\mathcal{C}$ consists either of (i) conjunctions of $k$ literals with at most one negated each, or (ii) conjunctions of $k$ literals with at most one positive each, or (iii) conjunctions of 2 literals, or (iv) XORs of $k$ literals. In all these cases, the $\mathcal{C}$-DL isomorphism problem is reducible to GI. Moreover, when $\mathcal{C}$ consists of XORs of two literals this isomorphism problem is in polynomial time. We will refer to this last case as $2 \oplus$-DL. We have shown that Graph Isomorphism is reducible to $\mathcal{C}$-DL-Iso when $\mathcal{C}$ is any of the above four classes. For all other $\mathcal{C}$, by Lemma $1.3, \mathcal{C}$-DL-Iso is coNP-hard. This shows the Schaefer-type dichotomy for the isomorphism problem of decision lists.

Let $L$ be a $2 \oplus$-DL, i.e., $L$ is given by a sequence of pairs $\left(p_{i}, b_{i}\right)$ where $b_{i} \in\{0,1\}$ and each $p_{i}$ is an XOR of two literals. We say that a pair $\left(p_{i}, b_{i}\right)$ fires on an assignment $x$, if $i$ is the least index such that $p_{i}(x)=1$. Let $f_{L}$ denote the function computed by $L$.

We first construct a normal form representation for $2 \oplus$-DLs to obtain an equivalent decision list where the tuples are partitioned into the sets $B_{1}, \ldots, B_{m}$, 
where the second component of each pair in $B_{i}$ is 0 if $i$ is odd, and is 1 if $i$ is even, for all $i$ (in this normal form the set $B_{1}$ could possibly be empty). We then exploit the structure of the normal form and in polynomial time transform the $2 \oplus$-DL isomorphism problem to Tree Isomorphism which can be solved efficiently.

We first explain the normal form $N_{L}$ (which is also a $2 \oplus-\mathrm{DL}$ ) for a given $2 \oplus$-DL $L$. For each pair of literals $l_{i}$ and $l_{j}$, if $l_{i} \oplus l_{j}=1$ implies that the function value is 0 , we add $\left(l_{i} \oplus l_{j}, 0\right)$ to the set of pairs $B_{1}$ of $N_{L}$. We can find such pairs by replacing all occurrences of $l_{j}$ by $\overline{l_{i}}$ in the decision list and checking if it computes the constant function 0 . After $B_{1}$ is computed, we compute the set $B_{2}$ as follows: find literals $l_{r}$ and $l_{p}$ such that $\left(l_{p} \oplus l_{r}\right) \wedge \bigwedge_{p_{i} \in B_{1}} \neg p_{i} \Rightarrow f_{L}$. It is easy to see that such pairs can be found efficiently. For each such pair we include $\left(l_{r} \oplus l_{p}, 1\right)$ in $B_{2}$. Continuing this construction, we obtain sets of pairs $B_{1}, B_{2}, \ldots, B_{m}$. This $2 \oplus$-DL is the normal form $N_{L}$ for $L$. The following lemma summarizes this normal form construction.

Lemma 4.1. If $L$ is a $2 \oplus-D L$ then $N_{L}$ is a normal form representation for $L$. Moreover, $N_{L}$ is computable in time polynomial in $|L|$.

Let $L$ be a $2 \oplus$-DL and $N_{L}$ be its normal form. We will efficiently encode $N_{L}$ as a rooted tree $T_{L}$ so that the following holds: For two $2 \oplus$-DLs $L_{1}$ and $L_{2}$, $f_{L_{1}}$ and $f_{L_{2}}$ are isomorphic if and only if the trees $T_{L_{1}}$ and $T_{L_{2}}$ are isomorphic. Recall that the normal form $N_{L}$ consists of sets of pairs $B_{1}, B_{2}, \ldots, B_{m}$. Consider inputs for which none of the pairs $\left(l_{i} \oplus l_{j}, 0\right) \in B_{1}$ fire. For all such inputs $l_{i}=l_{j}$ holds for each pair $\left(l_{i} \oplus l_{j}, 0\right) \in B_{1}$. These equalities induces a partition on the set of all variables into subsets $A_{1, p}, A_{1, n} A_{2, p}, A_{2, n} \ldots, A_{k, p}, A_{k, n}$, so that on any input for which the decision list reaches $B_{2}$ and for each $\ell$, all variables in $A_{\ell, p}$ are equal and all variables in $A_{\ell, n}$ are equal and complementary to the variables in $A_{\ell, p}$. Let $A_{\ell}=A_{\ell, p} \cup A_{\ell, n}$. Notice that some of the $A_{\ell}$ could be singletons, e.g. when the corresponding literals do not occur in $B_{1}$, and some $A_{\ell, p}$ or $A_{\ell, n}$ could be empty.

Now, we construct a new $2 \oplus$-DL from $N_{L}$ as follows: Delete $B_{1}$ from $N_{L}$. Introduce a new variables $y_{\ell}, 1 \leq \ell \leq k$, and in the decision list $B_{2}, \ldots, B_{m}$ replace by the variable $y_{\ell}$ all occurrences of variables in $A_{\ell, p}$ and replace by $\overline{y_{\ell}}$ all occurrences of variables in $A_{\ell, n}$, for each $\ell$.

Let $\hat{L}=\left(\hat{B_{2}}, \ldots, \hat{B_{m}}\right)$ denote this new $2 \oplus$-DL defined on the new input variables $y_{\ell}, 1 \leq \ell \leq k$. Recursively, we obtain a rooted tree $T_{\hat{L}}$ with leaves labeled by $y_{\ell}, 1 \leq \ell \leq k$ corresponding to $\hat{L}$. Now, to obtain the rooted tree $T_{L}$ from $T_{\hat{L}}$, we insert two children $(\ell, p)$ and $(\ell, n)$ to the leaf labeled $y_{\ell}$, and make the elements of $A_{\ell, p}$ children of $(\ell, p)$ and the elements of $A_{\ell, n}$ children of $(\ell, n)$. To complete the construction note that the base case when $N_{L}$ consists of only $B_{1}$ is easy to handle (since it is a constant function we create a depth one rooted tree $T_{L}$ with one leaf for each variable).

Since $N_{L}$ is a normal form representation for $L$, for any permutation $\pi$ of the variables $x_{1}, x_{2}, \ldots, x_{n}$ we have $N_{L^{\pi}}=\left(N_{L}\right)^{\pi}$. Suppose $L_{1}$ and $L_{2}$ are $2 \oplus$ DLs computing isomorphic functions and $\pi$ is an isomorphism: $L_{1}^{\pi}$ and $L_{2}$ are equivalent functions. Now, since $N_{L_{1}}$ and $N_{L_{2}}$ are normal forms for $L_{1}$ and $L_{2}$ 
we have $\left(N_{L_{1}}\right)^{\pi}=N_{L_{1}^{\pi}}=N_{L_{2}}$. By the above construction it is easy to see that the rooted trees $T_{L_{1}}$ and $T_{L_{2}}$ are isomorphic via a permutation $\psi$ such that $\psi$ restricted to the leaves of $T_{L_{1}}$ is the permutation $\pi$ (indeed, $\psi$ is the unique extension of $\pi$ to the internal nodes of $T_{L_{1}}$ ). Conversely, if $T_{L_{1}}$ and $T_{L_{2}}$ are isomorphic via a permutation $\psi$ then, from our construction we can argue that $L_{1}$ and $L_{2}$ are isomorphic, where the isomorphism is given by $\psi$ restricted to the leaves of $T_{L_{1}}$. We summarize the above discussion in the following lemma.

Lemma 4.2. The Boolean functions computed by two $2 \oplus-D L s L_{1}$ and $L_{2}$ are isomorphic if and only if the rooted trees $T_{L_{1}}$ and $T_{L_{2}}$ are isomorphic. Furthermore, given an isomorphism from $T_{L_{1}}$ to $T_{L_{2}}$ we can recover in polynomial time an isomorphism from $f_{L_{1}}$ to $f_{L_{2}}$.

Since testing isomorphism between trees can be done efficiently, we have the following theorem.

Theorem 4.3. The $2 \oplus-D L$ isomorphism problem is in polynomial time.

We now turn to the remaining variants of the $\mathcal{C}$-DL isomorphism problem. A $\mathcal{C}$-DL is a list of pairs $\left(C\left(x_{i_{1}}, \ldots, x_{i_{r}}\right), b\right)$, where $r$ is some fixed constant and $C\left(x_{i_{1}}, \ldots, x_{i_{r}}\right)$ is a $\mathcal{C}$-term. We will consider $\mathcal{C}$ to be one of the following classes of functions: (i) The $\mathcal{C}$-terms are of the form $l_{i} \wedge l_{j}$. (ii) The $\mathcal{C}$-terms are of the form $l_{i_{1}} \wedge \cdots \wedge l_{i_{r}}$, where $r$ is a fixed constant and at most one literal is positive. (iii) The $\mathcal{C}$-terms are of the form $l_{i_{1}} \wedge \cdots \wedge l_{i_{r}}$, where $r$ is a fixed constant and at most one literal is negative. (iv) The $\mathcal{C}$-terms are of the form $l_{i_{1}} \oplus \cdots \oplus l_{i_{r}}$, where $r \geq 3$.

We show that in all these cases $\mathcal{C}$-DL Isomorphism is reducible to Graph Isomorphism.

Theorem 4.4. The $\mathcal{C}-D L$ isomorphism problem, where $\mathcal{C}$ is one of the function classes above, is polynomial-time reducible to Graph Isomorphism.

We give a reduction from $\mathcal{C}$-DL-Iso to the label-respecting isomorphism problem of labeled trees which is equivalent to Graph Isomorphism RZ00. In this problem, we are given two rooted trees and additionally each vertex has a label. We ask if there is an isomorphism between the trees which is labelrespecting. I.e. if two vertices in the first tree have the same label, their images in the second tree have the same label. An equivalent generalized version is finding isomorphism of colored labeled trees, in which each vertex also has a color and we ask for color-preserving, label-respecting isomorphism.

Given a $\mathcal{C}$-DL $L$ on variables $\left\{x_{1}, \ldots, x_{n}\right\}$, we compute a normal form in polynomial time for the associated Boolean function $f_{L}$.

We find all $r$-tuples of literals, $T_{1}$, such that setting the associated $\mathcal{C}$-term to true forces $f_{L}$ to be constant. In general, we find all $r$-tuples of literals, $T_{i}$, such that setting the associated $\mathcal{C}$-term to true given the premise that all the $\mathcal{C}$-terms in $T_{j}, j<i$ are false forces $f_{L}$ to be constant. For the cases of $\mathcal{C}$ we are considering, checking this amounts to solving either 2CNF or Horn formula or Anti-Horn formula satisfiability or solving linear equations modulo 2. 
Such a process yields a sequence $T_{1}, \ldots, T_{m}$ of sets of $r$-tuples of literals which partitions all (the at most $(2 n)^{r}$ many) $r$-tuples of literals. As we saw for $2 \oplus$-DLs, this sequence $T_{1}, \ldots, T_{m}$ actually yields a $\mathcal{C}$-DL which is a normal form representation for $L$.

We will now encode $N_{L}$ as a labeled tree $T_{L}$ (in the sense of [RZ00]). It turns out that two $\mathcal{C}$-DLs $L_{1}$ and $L_{2}$ compute isomorphic functions if and only if there is a label-respecting tree isomorphism from $T_{L_{1}}$ to $T_{L_{2}}$. We outline the encoding algorithm which takes $N_{L}$ as input and computes a labeled tree $T_{L}$ : Let $T_{1}, T_{2}, \ldots, T_{m}$ be the $r$-tuple sets defining $N_{L}$. We create a root node with $m$ children corresponding to $T_{1}, T_{2}, \ldots, T_{m}$, where the node for $T_{i}$ is colored $i$. In the subtree rooted at the node corresponding to $T_{i}$ we create a child $c$ for each $r$-tuple $C \in T_{i}$. The node $c$ will have $r$ children which are leaves labeled by the corresponding variable name (in $x_{1}, x_{2}, \ldots, x_{n}$ ) and colored $p$ or $n$ depending on whether that literal occurring in $C$ is positive or negative.

This completes the construction of the labeled tree $T_{L}$. It is easy to verify that if the Boolean functions computed by $L_{1}$ and $L_{2}$ are isomorphic via a permutation $\pi$ then, in fact, $\pi$ acting on the leaf labels of $T_{L_{1}}$ induces an isomorphism from $T_{L_{1}}$ to $T_{L_{2}}$. Conversely, if there is a label-respecting isomorphism $\psi$ from $T_{L_{1}}$ to $T_{L_{2}}$, then $\psi$ induces a permutation $\pi$ on the leaf labels of $T_{L_{1}}$ which turns out to be an isomorphism from $f_{L_{1}}$ to $f_{L_{2}}$. This completes the proof sketch of Theorem 4.4.

\section{References}

[ADKK12] V. Arvind, Bireswar Das, Johannes Köbler, and Sebastian Kuhnert, The isomorphism problem for $k$-trees is complete for logspace, Inf. Comput. 217 (2012), 1-11.

[AT96] Manindra Agrawal and Thomas Thierauf, The Boolean isomorphism problem, FOCS, 1996, pp. 422-430.

[BC08] László Babai and Paolo Codenotti, Isomorhism of hypergraphs of low rank in moderately exponential time, FOCS, 2008, pp. 667-676.

[BHRV04] Elmar Böhler, Edith Hemaspaandra, Steffen Reith, and Heribert Vollmer, The complexity of Boolean constraint isomorphism, STACS, 2004, pp. 164175.

[BL83] László Babai and Eugene M. Luks, Canonical labeling of graphs, STOC, 1983, pp. 171-183.

[EH89] Andrzej Ehrenfeucht and David Haussler, Learning decision trees from random examples, Inf. Comput. 82 (1989), no. 3, 231-246.

[Luk99] Eugene M. Luks, Hypergraph isomorphism and structural equivalence of Boolean functions, STOC, 1999, pp. 652-658.

[Riv87] Ronald L. Rivest, Learning decision lists, Machine Learning 2 (1987), no. 3, 229-246.

[RZ00] Sarnath Ramnath and Peiyi Zhao, On the isomorphism of expressions, Inf. Process. Lett. 74 (2000), no. 3-4, 97-102.

[Sch78] Thomas J. Schaefer, The complexity of satisfiability problems, STOC, 1978, pp. 216-226.

[Thi00] Thomas Thierauf, The computational complexity of equivalence and isomorphism problems, LNCS, vol. 1852, Springer, 2000. 